Brit. J. industr. Med., 1953, 10, 241.

\title{
AIRBORNE DUST IN COAL MINES: THE SAMPLING PROBLEM
}

\author{
BY \\ W. M. LONG \\ From the Central Research Establishment I, National Coal Board
}

(RECEIVED FOR PUBLICATION MARCH 5, 1953)

Dust sampling has played a very important part in the drive against dust in British mines. It is becoming widely realized, however, that the present sampling procedures and instruments are far from satisfactory. Opinions tend to vary on what changes should be made, and the following survey of the problem attempts to reconcile some of the points of difference.

The scene of operations is the coal face, a long tunnel through which a stream of air is drawn; dust is produced locally by a variety of actions ranging from mechanical coal cutting to the impact of boots on the floor, and the problem is to measure the dust hazard to the men working in the tunnel.

It seems useful at the outset to distinguish two main sampling functions, research and routine control. In the research category falls the general task of tracking down the dust, of finding which pits are dusty, where suppression is needed, etc. Routine control is concerned with keeping watch on the dust levels, and giving warning of any dangerous changes. At the moment the main emphasis is on research, but routine control will soon become predominant.

There seems little doubt that for routine dust control a scheme on the lines of the quality control systems of the manufacturing industries represents the only really satisfactory solution. It is sometimes held that a system of routine inspection at regular intervals, say every three months, would be sufficient. But conditions on a coal face are not static; as work progresses they continually vary, and a single survey forms an inadequate basis for pronouncing a face safe for any extended period.

The variability of the dust concentrations is in fact considerable. The experience of the Pneumoconiosis Research Unit (Oldham and Roach, 1952) indicates that with the instruments at present available it is sometimes necessary to continue sampling for more than a week before the "true" average dust concentration can be located within $50 \%$ of its value with a reasonable degree of confidence. (In his 1953 paper (p. 227) Oldham is concerned with the accuracy of estimation of the mean concentration for any one shift, and for various reasons does not take into account day-today variation ; the latter, however, must be considered in any final appraisal of the accuracy of sampling.) These findings refer to sampling with the thermal precipitator, the most accurate of the instruments in current use. Incidentally they bring out very clearly the impossibility of the task which confronts the mining inspectors, who are required to assess conditions on a face from a single brief survey, usually covering only one shift.

An accuracy of $\pm 50 \%$ reached after a week's sampling, or about $7 \%$ after a year, is not impressive ; nevertheless, it would probably suffice, for the onset and subsequent progress of pneumoconiosis of coal miners is very slow. It seems clear from the survey of South Wales miners by D'Arcy Hart and Aslett (1942) that even heavy concentrations of anthracite dust normally take at least five years to produce detectable changes. Clearly in routine control sampling only long-term variations are important, and no sample which relates to a period of less than a week is of any real value. Looked at from this point of view, the great disadvantage of current instruments is that they are grossly uneconomic: the thermal precipitator, for example, collects its sample over a period of a few minutes and each sample is evaluated by counting individual particles. The amount of skilled labour which must be expended to collect and assess a meaningful sample is prodigious. Notwithstanding the good work which has been done with existing instruments, they are very wasteful and the industry cannot afford them.

What is needed is an instrument which will run 
for a period of at least a week with the minimum of attention: that is, a continuously operating, automatic sampler. There does not seem to be any compelling reason why a successful instrument of this type should not be developed. A choice of measuring principles is available, as shown by the diversity of instruments which have been used at one time or another. There have, it is true, been differences of opinion over the merits of different units in which dust concentration can be expressed (numbers of particles per c.c., projected area, etc.), but a great deal more will have to be known about the disease before it can be decided which unit is most appropriate ; in any case it has been shown (Bedford and Warner, 1943; Wynn and Davies, 1951) that most of the relevant physical characteristics are highly correlated in the narrow size range involved (particle diameter $\frac{1}{2}$ or 1 to 5 microns). What is needed is a cheap, simple and reliable instrument which will distinguish in an ordered and consistent fashion between different levels of dustiness, and the question of the units in which the answer is expressed is largely irrelevant.

Equally unnecessary are disagreements over whether the particle size range should be defined in terms of the projected area of the particles or by the settling velocity, that is, whether the sizes of the particles to be included in the sample should be judged by eye or by an elutriator. However variable the relation between the two properties may be in individual particles, with a substantial number spread over a five or ten to one size range, a high correlation between the results they give must be expected ; and in fact recent trials by the National Coal Board have shown that particle counts obtained by the two methods are practically identical. The choice can be made on the grounds of convenience in instrument design.

The use of automatic samplers brings up another point on which opinions differ, depending on which of two views on the probable relation of quantity of dust to disease is taken. One view is that the total amount of dust collected in the lungs is the important factor determining the severity of disease, and on this basis the average concentration over the whole of the period throughout which the men are exposed is the appropriate measure of the hazard. The other, slightly more complex, is that the higher concentrations are disproportionately important; that short exposure to high concentrations is more harmful than long exposure to low ones. The influence of this second theory makes itself felt in regulations which require that sampling should be confined to periods when the dustiness is at a maximum. Adherence to a rule of this kind would certainly hinder the production of an automatic instrument. It would also make it difficult to obtain uniformity of sampling practice and comparability of results, since conditions vary from one pit to another and " period of maximum dustiness" does not always have the same meaning. The requirement is in any case unnecessary. Suppose the second or " overwhelming concentration" theory about the disease is accepted, then the index of dustiness used must reflect the frequency of occurrence, the duration, and the magnitude of those concentrations whose magnitude exceeds some critical value. It must also be a statistical index : a mere record of particulars of individual dust " peaks" over periods of time long enough to be of any consequence, would be hopelessly indigestible. Any such index must be a function of the parameters of the distribution of dustiness over the period. In practice, a convenient and sufficiently accurate way of summarizing the relevant properties of the distribution would be to quote its first two moments ; in other words, its mean and standard deviation. But, as it happens, these distributions are of the type in which the standard deviation is predominantly a function of the mean itself, and the standard deviation increases with the mean. So the mean concentration is a valid index of the hazard under the second hypothesis as well as the first. (For a further exploration of this point, see Oldham, 1953.) It would be a relatively simple matter to fix a "permissible level" of the mean concentration which would ensure that the critical concentration (at which the dust was assumed to become harmful) would not be exceeded more than a given small proportion (say $5 \%$ or $1 \%$ ) of the time.

Quite apart from the question of its theoretical validity the use of the mean concentration would greatly ease the development of a successful automatic instrument, for obvious reasons. A number of subsidiary tasks in the maintenance of dust control would also be simplified. Indeed there is every indication that the adoption of a more complex criterion would prove a costly strategic mistake.

There remains the spatial problem : where the instrument should be placed, and whether it should be stationary or mobile. It has often been said that the ideal would be to sample the air which each collier breathes, but it seems that this is unlikely to be achieved within a reasonable space of time and that we must therefore be content with sampling a more general body of air, relying on the high correlation which must exist between the dustiness of the environment and the dustiness of the air which the collier actually breathes. A good 
correlation between the "approximate" and the " exact" measurements is quite sufficient for control purposes, and even if exact measurements could be obtained it is unlikely that they would be worth the trouble involved - each collier would have to breathe through a sampling instrument resembling a gas-mask.

The basic element in the spatial problem is the choice between "fixed-point" and " roving" sampling, that is, between keeping the instrument at one position on the face, and moving it up and down the face. The roving principle at first sight seems an obvious one to adopt, but from the control sampling standpoint it possesses serious disadvantages. The axiomatic requirement of control sampling is that the men working in the worst conditions on the face should be protected (the conditions being assessed over a reasonable period of time, since we are not interested in short-term fluctuations). Only the information necessary for achieving this need be collected. The type of roving sample which has been used so far is one in which short-period samples are taken at various points along the face during the working shift, the underlying assumptions being that the location of the maximum dust concentration changes from time to time and that by moving the instrument up and down the face one can ensure that the worst conditions are evaluated. But it seems likely that the first assumption is true only if the measurements are confined to short intervals of time : the location of the maximum concentration may fluctuate from minute to minute or even hour to hour, but when measured over reasonable periods of time it is in general quite stable; and, as argued above, it is the long-term measurements which matter. Even if this reasoning is rejected, the information we now possess invalidates the assumption that the face can be adequately covered with one or two instruments; the samples are too small and the variability of the dust concentrations too high.

The current type of roving sample begins to have value only when used to obtain an estimate of the average concentration on the face, and it is the right type for this purpose. ("Average" in this context refers of course to the distribution in space, not in time.) But for control sampling purposes, since we are interested not in the average but in the maximum, most of the information provided by the roving sample is irrelevant.

It has been suggested that the "random collier" method described by Oldham and Roach (1952) could be used for control sampling. In this method the sampling instrument is associated with a collier chosen at random (more than one instrument may be used, the number of colliers being correspondingly increased). In control sampling, the collier would retain his instrument for a specified period, when a fresh random allocation would be made, and this redeployment of instruments would be repeated at regular intervals. It will be seen that the scheme is essentially of the roving sample type, and is open to precisely the same charges of irrelevancy and inadequacy. For control sampling purposes it does not suffice to show that " random " or " average" conditions on the face conform to the standards.

The fixed point system is clearly the one to use, if we can predict where the worst dust conditions will occur (the conditions being assessed over reasonable periods of time, since we are not interested in short-term fluctuations). The information collected is all relevant and is fully used, in contrast with that furnished by the roving sample. Indeed it would scarcely be an exaggeration to say, in view of the difficulty of getting a sufficiently reliable measure of concentration, that is, of collecting sufficient information, that the only hope of establishing effective control in the dustier pits lies in the possibility of applying the fixed point method. It must be regarded as fortunate, therefore, that the worst conditions nearly always do seem to occur at the same place, namely, at the return end of the face. The harmful fraction of the dust has such a low settling velocity that the amount lost from the airstream by deposition on the walls and floor of the tunnel is small enough to be neglected ; nearly all the harmful dust raised on the face eventually finds its way to the return end. The concentration of harmful dust normally increases, on the average, from the intake end to the return end, as the air in its path passes more and more dust sources. (Indeed it has been observed by the N.C.B. scientific control staff that the concentration of all airborne dust-including the innocuous heavier fractionoften shows the same tendency.) The appropriate position for the sampling instrument would therefore seem to be at the return end of the face, or perhaps (in single-unit faces) in the return airway itself. This method is of course in fairly common use nowadays, and seems to be a convenient one in practice.

The objection to return-end sampling is that the reasoning underlying it is conceived in terms of average concentrations across the cross-sections of the face, and that men who are working near a source of dust may be exposed to conditions worse than the average in the cross-section at that point, and possibly worse than those in the return end. There are, however, circumstances which tend to mitigate this objection. The collier 
does not normally breathe the dust which he himself raises, but that raised by his workmates further up the air stream, and the turbulence produced in the air stream by obstacles such as props and by the movements of the men themselves, tends to even out the dust concentration across the cross section. Moreover the high concentrations of harmful dust which arise near a dust source are associated with clouds of coarse dust which are physically irritating and which are avoided if possible. And, of course, if the collier moves to and fro across the air stream at any point the average concentration to which he is exposed tends to become equal to the average concentration across the cross-section at that point.

However, the question is not disposed of and experimental investigation is indicated. If indeed it should turn out that some men are exposed to conditions worse than those obtaining at the return end of the face, a possible remedy might be to adjust the level of dustiness permitted at the return end to some value which would ensure that conditions on the rest of the face were satisfactory. Otherwise a suitable position nearer to the dust source would have to be sought.

In sampling for research purposes, no hard and fast rules can be laid down. It is certain, however, that a continuously operating automatic instrument would be of immense value, especially if it were readily portable. The need for such an instrument for work in the field of $x$-ray examination has already been noted by Cochrane, Fletcher, Gilson, and Hugh-Jones (1951).

Extensive application of the quality control scheme mentioned above would in time afford a by no means negligible increase in our knowledge of the environmental conditions associated with dust disease. But " conveyor-belt" statistics of the kind this system would produce usually give only broad indications of trends, points for further study, etc., and for detailed investigations the "random collier" method, which is based on modern principles of experimental design, has, as Oldham and Roach show, several important advantages.

This paper is published by permission of the Director General, Scientific Department, National Coal Board. The views expressed in it are those of the author, and must not necessarily be taken to have the Board's support.

\section{REFERENCES}

Cochrane, A. L., Fletcher, C. M., Gilson, J. C., and Hugh-Jones, P. (1951). British Journal of Industrial Medicine, 8, 53.

D'Arcy Hart, P., and Aslett, E. A. (1942). Med. Res. Coun. Spec. Rep. Ser. No. 243, pp. 53, 64, 90-93.

Bedford, T., and Warner, C. G. (1943). Med. Res. Counc. Spec. Rep. Ser. No. 244, pp. 13-15.

Oldham, P. D. (1953). British Journal of Industrial Medicine, 10, 227. Oldham, P. D., and Roach, S. A. (1952). Ibid., 9, 112.

Wynn, A. H. A., and Davies, J. G. (1951). S.M.R.E. Research Report No. 28 .

\section{Scholarships in Aid of Scientific Research}

The Council of the British Medical Association is prepared to receive applications for research scholarships, as follows :

An Ernest Hart Memorial Scholarship of the value of $£ 250$

A Walter Dixon Scholarship of the value of $£ 250$

These scholarships are given to candidates recommended by the Science Committee of the Association as qualified to undertake research in any subject (including State medicine) relating to the causation, prevention, or treatment of disease.

Each scholarship is tenable for one year, commencing on October 1, 1954. A current scholar may apply to be reappointed for a further year. No scholarship can be held for more than three years. A scholar is not necessarily required to devote the whole of his or her time to the work of research, but may be a member of H.M. Forces or may hold a junior appointment at a university, medical school, or hospital, provided the duties of such appointment will not, in the opinion of the Science Committee, interfere with his or her work as a scholar.

Applications for scholarships must be made not later than March 1, 1954, on the prescribed form, a copy of which will be supplied by me on application.

Applicants are required to furnish the names of three referees who are competent to speak as to their capacity or the research contemplated.

British Medical Association House,

A. Macrae, Secretary.

Tavistock Square, London, W.C.1. 